\title{
Adsorption sites and rotational tunneling of methyl groups in cubic I methyl fluoride water clathrate $\dagger$
}

\author{
M. Prager, ${ }^{a}$ J. Baumert, ${ }^{b}$ W. Press, ${ }^{b}$ M. Plazanet, ${ }^{b}$ J. S. Tse ${ }^{c}$ and D. D. Klug ${ }^{c}$ \\ ${ }^{a}$ Institut für Festkörperforschung, Forschungzentrum Jülich, D-52425 Jülich, Germany \\ ${ }^{\boldsymbol{b}}$ Institut Laue-Langevin, F-38042 Grenoble-Cedex, France \\ ${ }^{c}$ Steacie Institute of Molecular Sciences, National Research Council of Canada, Ottawa, \\ ON K1A OR6, Canada
}

Received 17th September 2004, Accepted 6th January 2005

First published as an Advance Article on the web 21st January 2005

\begin{abstract}
Neutron spectroscopy in the $\mu \mathrm{eV}$ and $\mathrm{meV}$ regime and quasielastic scattering is applied to characterize the dynamics of methyl groups of methyl fluoride guest molecules in cubic I $\mathrm{CH}_{3} \mathrm{~F}$-water clathrate. Only above $T \sim 60 \mathrm{~K}$ quasielastic spectra are unaffected by quantum effects. They are well described by two Lorentzians representing the $\mathrm{CH}_{3} \mathrm{~F}$ species in the small and large cages of the structure. The intensities show that both cages are completely filled. The linear broadenings with temperature follow the model of rotational diffusion. Two clearly separated tunneling bands were observed at $T=4.2 \mathrm{~K}$ and are also assigned to the two types of water cages. Disorder of the environment (H-bonds) is reflected in the shape of the bands. For the less hindered species housing the large cages the tunneling band can be quantitatively converted into a potential distribution function within the model of single particle rotation. Transitions to excited rotational states show the dominance of a sixfold potential term $\bar{V}_{6}=13 \mathrm{meV}$ modified by a weak threefold term distributed around a characteristic value $\bar{V}_{3}=0.9 \mathrm{meV}$. The potential distribution of $V_{3}$ influences the barrier for classical reorientation only weakly in agreement with the results from quasielastic data. Adsorption sites with the guest molecules oriented towards a hydrogen bond along one of twelve local twofold axes of the cage are proposed. Such sites are consistent with the sixfold rotational potential and earlier results from methyl iodide clathrate. Rotation-translation coupling as an alternative dynamical process is excluded.
\end{abstract}

\section{Introduction}

Partly due to the discovery of methane clathrates as one of the largest energy reservoirs on earth ${ }^{1}$ inclusion compounds have newly gained the interest of the public and the scientific community. The material became commonly known as 'burning ice'. Beside such natural systems chemists are able to combine many different host and guest molecules into a fascinating variety of inclusion compounds. ${ }^{2-4}$ A nearly universal versatility in the choice of guest molecules may allow to enclathrate biologically interesting substances for which the ordered water cage may be considered as a model hydration shell. ${ }^{5}$

If the host lattice is composed of water the compounds are called clathrates. ${ }^{3}$ The water cages are stabilized by the presence of the guest molecules. They are formed from pentagonal, hexagonal and square rings of water molecules connected at their edges and are characterized by the number and type of polygons forming the cage. The simplest cage consisting of 12 pentagonal faces is named the pentagon dodecahedron, abbreviated $5^{12}$. Methane clathrate crystallizes in the cubic I structure with space group $P m 3 n$ with a unit cell formed by six $5^{12} 6^{2}$ and two $5^{12}$ cages. $^{6,7}$ Larger guest molecules require larger cages and lead to different crystal structures. Incorporation of methyl iodide clathrate for example leads to the cubic II structure with eight $5^{12} 6^{4}$ and sixteen $5^{12}$ water cages. $^{8}$

The dynamics of methane in the natural clathrate shows fundamentally interesting behavior. The large size of the cages allows for very anharmonic soft translational oscillations

$\dagger$ Presented at the 7th International Conference on Quasi-elastic Neutron Scattering, Arcachon, France, September 1-4, 2004. (rattling) and almost free rotation. At the lowest temperature the molecular motion was quantitatively described as single particle quantum rotation ${ }^{9}$ in weak rotational potentials which differ slightly from cage to cage due to different arrangements of the hydrogen bonds of the cage surface. ${ }^{10}$

Guided by the simplicity of $1 \mathrm{D}$ rotation we became interested in clathrates housing guest molecules containing methyl groups. They were expected to show quantum rotational dynamics of the $\mathrm{CH}_{3}$ groups analoguously to the methane case. The spectra of $1 \mathrm{D}$ rotors can be analysed much more directly than the 3D dynamics of methane molecules. Indeed, new information on the complex potential surface of the only populated $5^{12} 6^{4}$ cage could be obtained in the case of methyl iodide clathrate. ${ }^{8}$ Three separated but overlapping tunneling bands were assigned to three different adsorption sites. Completely new information on the hydrogen bond dynamics on the cage surface were deduced by correlating the adsorption sites with the site dependent spin conversion times. This study shall now be extended to methyl fluoride clathrate. The smaller guest molecules cause the host lattice to crystallize like methane clathrate in the cubic I structure with space group $P m 3 n$ and lattice parameter $a \sim 11.8 \AA$. As in methane clathrate large and small water cages are filled by guest molecules. The large cage of this structure is $5^{12} 6^{2}$ and is shown by Fig. 1. The location of the hexagons on opposite sides of the cage leads to a local but not a crystallographic sixfold symmetry. In the cubic II structure of methyl iodide clathrate the four hexagons of the larger $5^{12} 6^{4}$ cages show a tetrahedral symmetry. Finally, the intermolecular interaction between host and guest molecules is changed. Fluorine is known in many systems to develop hydrogen bonds which may lead to new dynamic behavior. 


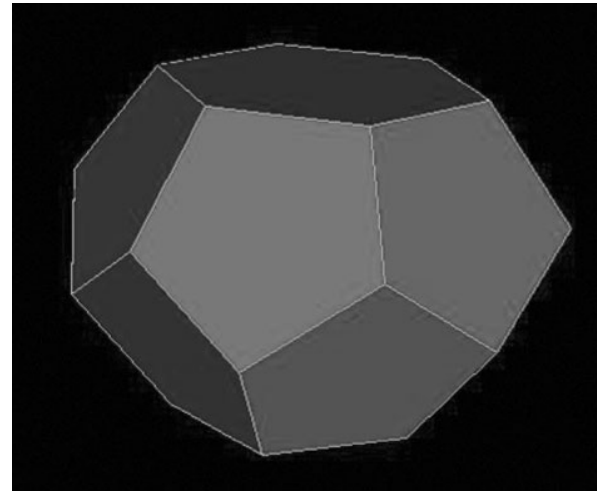

Fig. 1 The large $5^{12} 6^{2}$ cage of the methyl fluoride clathrate $\mathrm{CH}_{3} \mathrm{~F} \times$ $5.75 \mathrm{H}_{2} \mathrm{O}$, which contains the majority of $\mathrm{CH}_{3} \mathrm{~F}$ guest molecules. Each corner represents an oxygen of the water shell, each edge a hydrogen bond. There are inequivalent hydrogen bonds: 12 edges connect a pentagon with a hexagon, 12 connect 2 pentagons while on each side there is another pentagon, 12 connect 2 pentagons with a pentagon on one and a hexagon on the other side.

\section{Theory}

Two different models will be used to discuss the spectra, the model of single particle rotation (SPM) and rotation translation coupling (RTC).

\subsection{Single particle rotation}

The standard description of rotational tunneling and quasielastic scattering is the SPM. The environment of the $\mathrm{CH}_{3}$ group is represented by a rotational potential $V(\varphi)$. Due to the symmetry of the methyl group it is expressed in the form of a Fourier expansion of threefold symmetry up to order $N$

$$
V(\varphi)=\sum_{n=1}^{N} \frac{V_{3 n}}{2}\left(1-\cos \left(3 n \varphi-\varphi_{3 n}\right)\right)
$$

At low temperatures when the classical rotational jump dynamics is frozen this potential determines the excitations of the hindered quantum rotor as eigenvalues $E_{\mathrm{i}}$ of the stationary single particle Schrödinger equation ${ }^{9}$

$$
\left\{-B \frac{\partial^{2}}{\partial \varphi^{2}}+V(\varphi)\right\} \Psi_{i}=E_{i} \Psi_{i}
$$

Here $B=\hbar^{2} / 2 \Theta=0.655 \mathrm{meV}$ is the rotational constant of the methyl group with the moment of inertia $\Theta$. The tunnel splitting is the difference between the two lowest levels $\hbar \omega_{\mathrm{t}}=$ $E_{1}-E_{0}$. The knowledge of further transitions to higher rotational states, $E_{\mathrm{j}}-E_{\mathrm{i}}$, must be known to refine the shape of the rotational potential.

High resolution neutron spectroscopy is an especially successful technique to observe the low energy transitions of weakly hindered rotors. For finite potential size especially the groundstate tunnel splitting $\hbar w_{\mathrm{t}}$ gives rise to a simple scattering function ${ }^{9,11}$

$$
\begin{aligned}
S(Q, \omega)= & \left(\frac{5}{3}+\frac{4}{3} j_{0}(Q d)\right) \delta(\omega)+\left(\frac{2}{3}-\frac{2}{3} j_{0}(Q d)\right)\{\delta(\omega \\
& \left.\left.+\omega_{t}\right)+\delta\left(\omega-\omega_{t}\right)\right\} .
\end{aligned}
$$

with momentum transfer $Q$ and jump distance $d . j_{0}$ is the spherical Bessel function of 0 -th order. In the case of $\mathrm{CH}_{4} \mathrm{II}$ it could be shown, ${ }^{12}$ that the $Q$-dependence of a free rotor is very similar to that of a hindered tunneling species.

Classical jump reorientation at higher temperatures is characterized by a jump rate $\tau \sim \frac{1}{\Gamma(T)}$ which for large barriers obeys usually an Arrhenius function with a characteristic activation energy $E_{\mathrm{a}} \cdot E_{\mathrm{a}}$ is calculated from eqns. (2) and (1). Stochastic reorientational jumps lead to quasielastic scattering with the neutron scattering function ${ }^{13}$

$$
\begin{gathered}
S(Q, \omega)=\left(1+2 j_{0}(Q d)\right) \delta(\omega)+2\left(1-j_{0}(Q d)\right) L(\Gamma, \omega) \\
L(\Gamma, \omega)=\frac{1}{\pi} \frac{\Gamma}{\Gamma^{2}+\omega^{2}} \\
\Gamma(T)=\Gamma_{0} \exp \left(-E_{\mathrm{a}} / k T\right) .
\end{gathered}
$$

Here the jump distance $d$ is again identical with the protonproton distance of the methyl group.

For weak or negligible barrier height the methyl dynamics is better described by rotational diffusion on a circle of radius $\rho=d / \sqrt{3}$. This motion involves angular displacements of any distance. Therefore the scattering function

$$
\begin{gathered}
S(Q, \omega)=A_{0}(Q \rho) \delta(\omega)+\sum_{n=1}^{\infty} A_{n}(Q \rho) L\left(\Gamma_{n}, \omega\right) \\
\Gamma_{n}(T)=n^{2} D_{\mathrm{R}}
\end{gathered}
$$

contains an infinite number of Lorentzians $L\left(\Gamma_{n}, \omega\right)$ with non analytical structure factors $A_{n}(Q \rho)$ and the rotational diffusion constant $D_{\mathrm{R}} \cdot D_{\mathrm{R}}$ increases proportional to temperature $k T$. For details see refs. 9, 13 and 14. At small momentum transfers $Q \leq 2 \AA^{-1}$ as in our experiment only the term $n=1$ has a measurable structure factor $A_{n} \ll A_{1}, n \geq 2$ and the scattering functions of jump rotation and rotational diffusion are formally identical. They differ mainly in the temperature dependence of the Lorentzian linewidth. This will be used below.

\subsection{Rotation translation coupling}

In an environment with lateral freedom a rotor may find a minimum energy path of rotation by correlated rotation and displacement of its centre-of-mass from the symmetry position. The corresponding motion is called rotation-translation coupling and was first solved for the case of Hofmann clathrates ${ }^{14 c}$ where ammonia groups represent a threefold rotor in a fourfold environment. The Hamiltonian which characterizes the problem is

$$
\begin{aligned}
H= & B \frac{\partial^{2}}{\partial \varphi^{2}}-B_{\text {com }}\left(\frac{\partial}{\partial \alpha}-\frac{\partial}{\partial \varphi}\right)^{2}+2 A_{1} \cos (3 \varphi+m \alpha) \\
& +2 A_{2} \cos (m \alpha)
\end{aligned}
$$

Here $B_{\text {com }}$ is the rotational constant of the whole molecule with respect to the centre of mass displaced from the centre of symmetry/rotation by a distance $R$. $R$ is determined by the two parameters $A_{1}$ and $A_{2}$ defining the 2D potential surface. $\alpha$ is the angle of rotation of the centre of mass, the enviromental symmetry is $m=4$. Despite our cages show twofold, fivefold or sixfold symmetries corresponding to adsorption sites facing a hydrogen bond or the centre of a pentagon or the centre of a hexagon of the water cage we use the known mathematics for qualitative tests. The modification of eqn. (6) for symmetries $m=2,5$ or 6 may be rather complex and requires a verification. This is a challenge for a theoretician.

\section{Experimental results and discussion}

All following data were obtained by neutron scattering. Instruments of the Institut Laue-Langevin, Grenoble, and of the Forschungszentrum Jülich were used.

\subsection{Sample preparation and characterization}

The sample was prepared at the National Research Council of Canada. A detailed description of the preparation procedure is found in the literature. ${ }^{14 d}$ Due to this procedure the sample may contain some amount of bulk methyl fluoride. This 
contamination was removed by warming up the sample to $T=$ $190 \mathrm{~K}$ after the cold transfer into the sample holder. The sample was kept at this temperature which is above the boiling point of methyl fluoride in an inert gas atmosphere for about $20 \mathrm{~min}$. Since the kinetics for hydrate decomposition is much slower than boiling off of $\mathrm{CH}_{3} \mathrm{~F}$ the procedure cleans the sample without damaging it.

As in the earlier publication ${ }^{8}$ the measured librational phonon density of states is used to prove the formation of the clathrate. Spectra are taken using the thermal time-of-flight spectrometer SV29 at Forschungszentrum Jülich ${ }^{14 e}$ at various incoming wavelengths. The most interesting energy range up to $20 \mathrm{meV}$ is shown in Fig. 2. The band around $10 \mathrm{meV}$ which is only found in clathrate samples and not in water ice is clearly visible. The librational phonon density of states is used here as an analytical tool only and shall not be discussed with respect to other better resolved spectra of related clathrates. ${ }^{15-17}$

A comparison of spectra from an original sample with one exposed to the outlined temperature treatment showed almost no differences. This means at first that already the original material contained only negligible amounts of pure $\mathrm{CH}_{3} \mathrm{~F}$. Secondly this result proves the conservation of the clathrate structure during the boil-off procedure.

\subsection{Single particle description}

In this section the data are discussed within the model of single particle rotation. ${ }^{9}$ The most overwhelming number of systems are successfully described by this concept. ${ }^{11}$

3.2.1. Analysis of the groundstate splitting and disorder. The most precise information about rotational potentials is contained in the tunnel splitting of the librational groundstate. The respective spectra were obtained at the Institut Laue-Langevin using the cold time-of-flight spectrometer IN $5^{18}$ at wavelengths $\lambda=4.5 \AA, \lambda=6.5 \AA$ and $\lambda=9.0 \AA$. The flat sample had a thickness of $0.5 \mathrm{~mm}$ and was oriented under $45^{\circ}$ to the beam.

A characteristic spectrum is shown in Fig. 3. The prominent peak has an almost triangular shape with a sharp edge at about $0.6 \mathrm{meV}$. The SPM Hamiltonian yields a sharp tunnel splitting for a given rotational potential. The broad triangular intensity distribution observed must therefore be due to the presence of different rotational potentials and environments. The high energy edge is very close to the rotational constant $B=0.655$ meV which may be called the tunnel splitting of a completely free rotor. The intensity is maximum close to this value. Thus, a majority of methyl groups feels only a very weak rotational

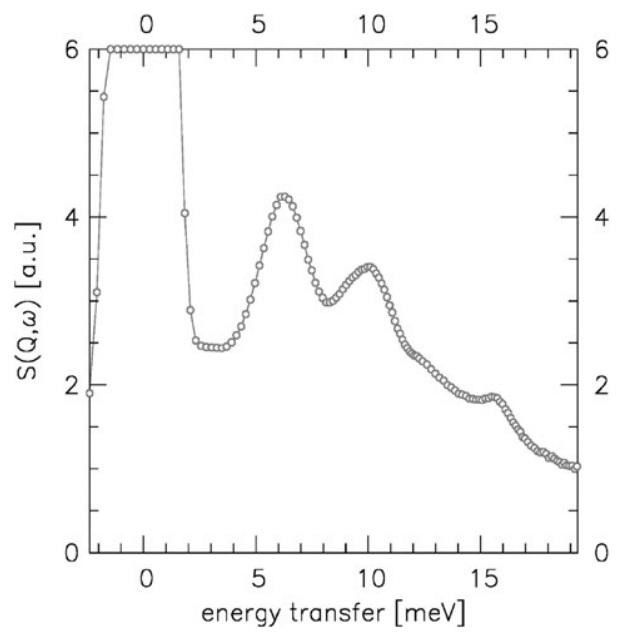

Fig. 2 Neutron scattering spectrum taken in the regime of lattice modes and summed over all scattering angles. Spectrometer: SV29 at Forschungszentrum Jülich. Wavelength $\lambda=1.81 \AA$. Sample temperature $T=4.2 \mathrm{~K}$. Average momentum transfer $Q=4.9 \AA^{-1}$.

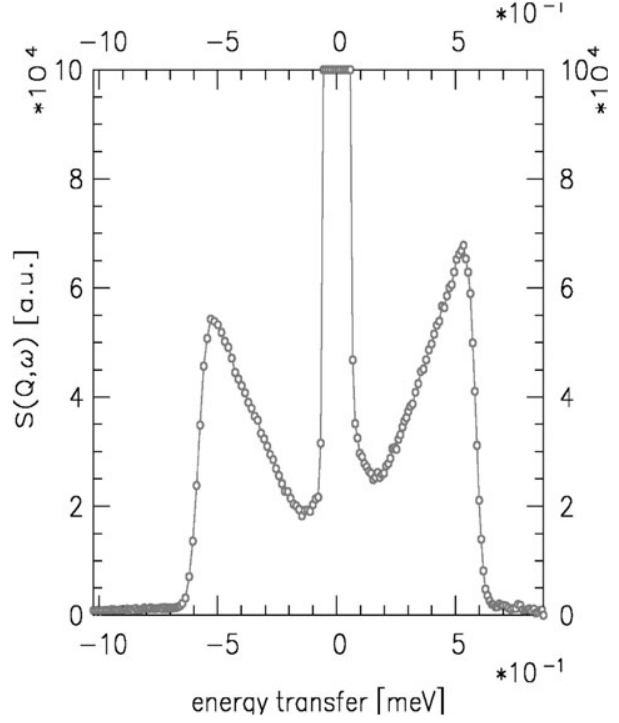

Fig. 3 Distribution of tunnel splitting of the methyl groundstate in methyl fluoride water clathrate. Fully protonated sample. Sample temperature $T=2.0 \mathrm{~K}$. Spectrometer: IN5 of ILL. Wavelength $\lambda=6.5 \AA$. Average momentum transfer $Q=1.6 \AA^{-1}$.

potential. From the maximum the intensity decays almost exactly proportional to the energy transfer $\hbar \omega: I=c \omega$. This means that the stronger the rotational barrier the rarer the corresponding environments.

The structure factors of the tunnel transitions are found to depend only very weakly on the shape and strength of the rotational potential. ${ }^{12}$ Thus, the intensity distribution of the tunneling band $I(\omega)$ can be converted ${ }^{19}$ into a potential distribution function $p(V)$ by

$$
p(V) \mathrm{d} V=I(\omega) \frac{\mathrm{d} \hbar \omega_{t}}{\mathrm{~d} V} \mathrm{~d} V
$$

The Schrödinger eqn. (2) of the SPM yields $\hbar \omega_{t}(V)$ and therefrom $\frac{\mathrm{d} \hbar \omega_{t}}{\mathrm{~d} V}$. Very often rotational potentials are described by two terms of the Fourier expansion eqn. (1). ${ }^{11}$ In this case the function $\hbar \omega_{t}(V)$ is represented by a 2D surface and a potential distribution function on this surface is characterized by four quantities, the most likely values $V_{30}$ and $V_{60}$ and corresponding spreads $\delta V_{30}$ and $\delta V_{60}$.

In previous work the analysis of disorder is restricted to pure $\cos (3 \varphi)$ potentials and Gaussian distributions. ${ }^{19,20}$ Hence only $V_{30}$ and $\delta V_{30}$ play a role. For this case $\hbar \omega_{t}\left(V_{3}\right)$ is given by the top thin solid line in Fig. 4 and delivers the derivative $\frac{\mathrm{d} \hbar \omega_{t}}{\mathrm{~d} V_{3}}$. Now we use the relation $\hbar \omega_{t}\left(V_{3}\right)$ a second time directly since the measured intensity profile is found to be $I \sim \omega$. We assume for simplicity that this relation is valid up to the free rotor case. The numerically resulting potential distribution function $p\left(V_{3}\right)$ is now given by the lower thick solid line of Fig. 4. Its maximum is at about $3 \mathrm{meV}$. At $V_{3}=11 \mathrm{meV}$ the probability $p\left(V_{3}\right)$ is already reduced to about $10 \%$ of the maximum. The exploitation of the measured intensity distribution makes us free from assumptions on the potential distribution function $p\left(V_{3}\right)$. The observed $p\left(V_{3}\right)$ is rather unique. The distribution found for methane clathrate in the experiment and by numerical modelling ${ }^{10}$ is Gaussian which is also the parametrization in the original paper. ${ }^{19}$

As we will see below the rotational potential is of dominant sixfold symmetry. While all qualitative arguments are still valid, the relation $\hbar \omega_{t}(V)$ and therewith the resulting potential distribution function change. The discussion of the dashed curves of Fig. 4 is postponed to Section 4 where we will include the effect of the potential shape on the potential distribution function by considering different cuts through the $2 \mathrm{D}$ surface $\hbar \omega_{t}\left(V_{3}, V_{6}\right)$. Especially the case of a constant sixfold potential term will be considered. 


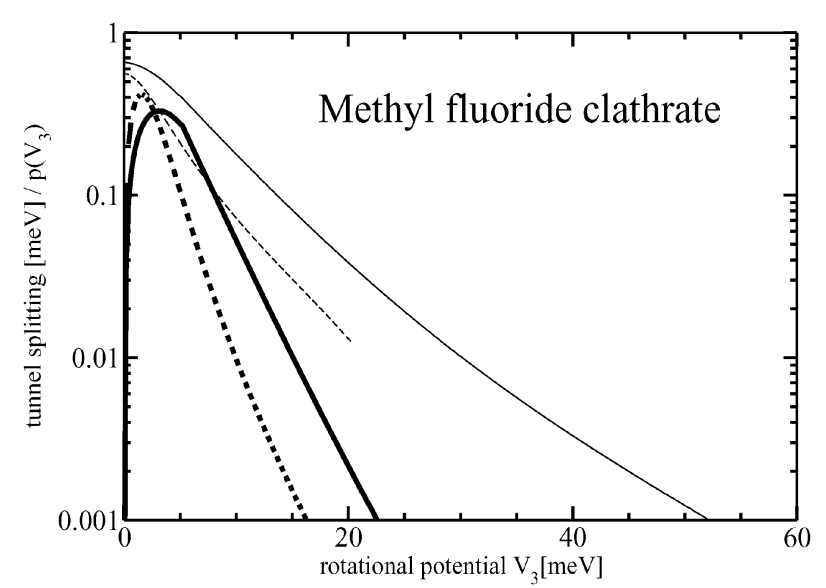

Fig. 4 Thin lines: theoretical changes of the tunnel splittings with the potential strength $V_{3}$. Solid line: $V_{3}=0.0 \mathrm{meV}$; dashed line: $V_{6}=13.0$ $\mathrm{meV}$. Corresponding potential distribution functions (thick lines) based on the observed linear decrease of tunneling intensity with tunneling energy.

Already in Fig. 3 but much clearer in the spectrum taken at a wavelength $\lambda=9.0 \AA$ A (Fig. 5) a wing of inelastic intensity close to the elastic line is observed. Since classical stochastic motion has died out at $T=2 \mathrm{~K}$ we assign this intensity to a second quantum rotation related with a clearly different adsorption site. The solid line in the figure shows a fit of this part of the spectrum by a Gaussian intensity distribution. Due to a missing analytical description of the broad triangular band the intensities of the two distributions are compared numerically. Their ratio is roughly 3 . This is the ratio of the number of large to small cages in the cubic I structure. Guided by the intensities of the two tunneling bands we assign the intense triangular band of little hindered rotors with guest molecules in the large cages $(75 \%)$ of the cubic I structure and the weak band to its small cages $(25 \%)$. Within the accuracy of the data the small cages are completely filled by $\mathrm{CH}_{3} \mathrm{~F}$ guest molecules. This corresponds well to the case of the methane guest molecules with their similar size where the small cages are also all filled. ${ }^{10}$ With this property both materials are somehow an exception. While the large cages of the structure are in general completely filled by guest molecules, the smaller cages may be only partly filled to stabilize the lattice. Therefore clathrates often are named non stoichiometric compounds.

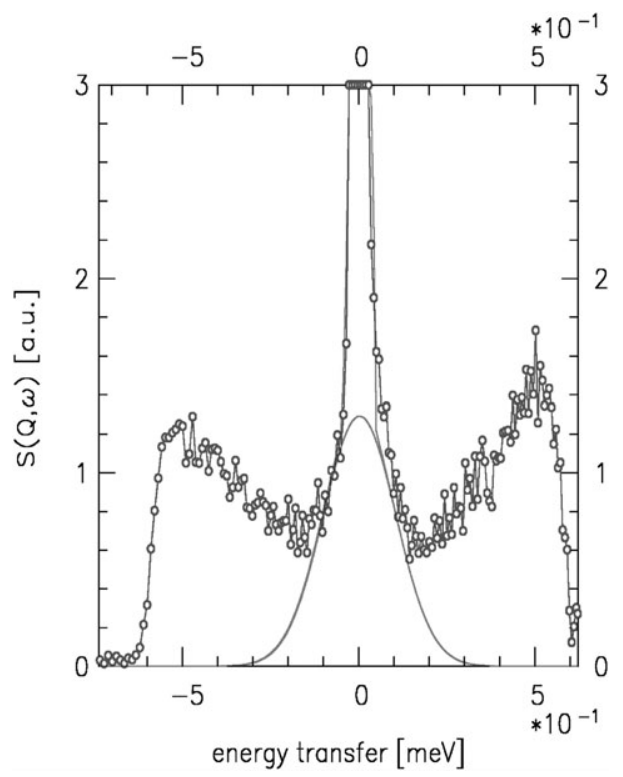

Fig. 5 Same as Fig. 3. Wavelength $\lambda=9.0 \AA$ Average momentum transfer $Q=1.1 \AA^{-1}$. The solid line models the narrow intensity distribution by a Gaussian of halfwidth $112 \mu \mathrm{eV}$.
Shorter intermolecular distances in the small cages increase the intermolecular interactions. As shown by pressure experiments this is in general connected with an increase of the rotational potentials. Methane clathrate is an example, however, where the small cages are described by similarly low barriers. ${ }^{10}$ A high local symmetry may counteract the effect of shorter distances to the wall. Mathematical modelling is needed to identify such behavior. Unfortunately the necessary structural information for such calculations, the location of the fluor atoms, are not yet available for our compound.

At the sample temperature of $T=2 \mathrm{~K}$ used in the experiment the excited tunneling state should be almost completely depopulated. The fact that we observe nearly the same intensity in energy gain as in energy loss shows that very long spin conversion times stabilize the excited tunneling $E$-state far from thermal equilibrium. With increasing sample temperature spin conversion becomes faster. The sample reaches thermal equilibrium only at temperatures characteristic of an intermediate situation between quantum mechanics and classical motion.

3.2.2 Transition from quantum mechanics to classics. The very interesting temperature regime where the quantum excitations transform into classical spectra according to the correspondance principle was only selectively explored. The main reason for this restriction is that the presence of disorder mixes in so many states that an evaluation of such spectra in the transition regime according to theory ${ }^{21}$ can not give clear and quantitative results. Fig. 6 shows two characteristic spectra. While at a sample temperature $T=30 \mathrm{~K}$ the inelasticity as a sign of quantum motion is still clearly visible in the broadened and shifted spectrum the data taken at $T=40 \mathrm{~K}$ look already much more quasielastic and require a closer look to detect the reminiscence of quantum character. Only above $T=50 \mathrm{~K}$ the classical regime seems to be reached. This temperature is found from fits with the classical scattering function. As with lowering the temperature an even weak inelasticity from quantum mechanics comes into play ( $c f$. Fig. 7) the quasielastic linewidths starts to stay constant or even to broaden.

3.2.3 Classical reorientation. Quasielastic spectra are shown in Fig. 8. It was possible to model quasielastic spectra by a single Lorentzian reasonably well. Based on the observation of two energetically separated species of methyl rotors the quasielastic spectra are modelled by two Lorentzians leading to

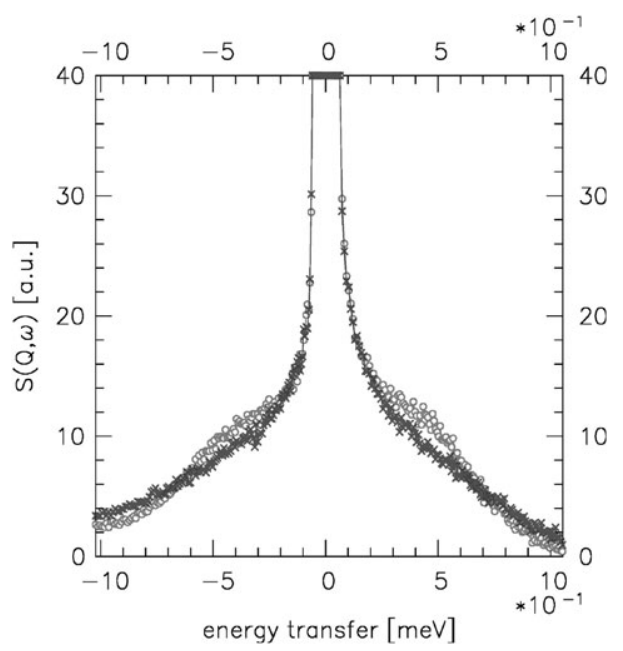

Fig. 6 Neutron spectra from the intermediate temperature regime between pure quantum mechanical excitations and purely stochastic reorientations. Sample temperatures $T=30 \mathrm{~K}(\square)$ and $T=40 \mathrm{~K}(\star)$. Spectrometer: IN5 at ILL. Wavelength $\lambda=6.5 \AA$. Sum over all scattering angles. Average momentum transfer $Q=1.9 \AA^{-1}$. 


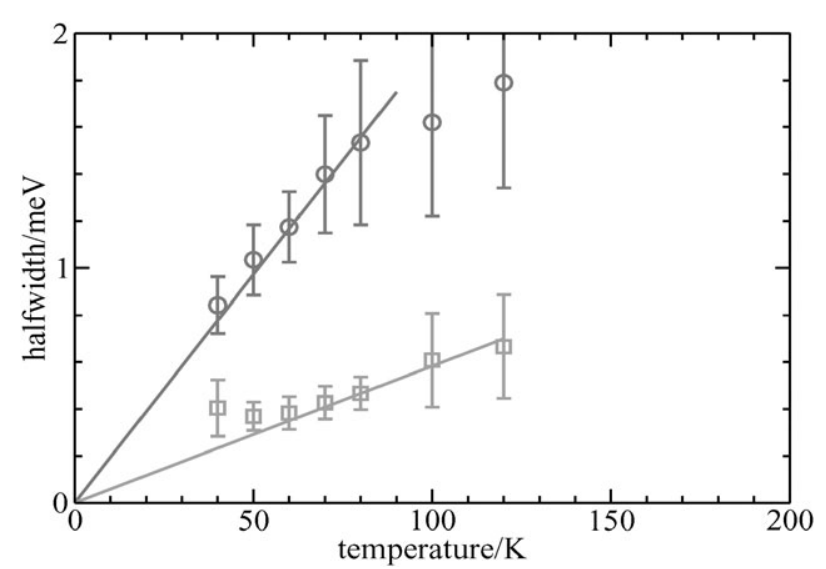

Fig. 7 Quasielastic linewidths of the two components (molecules in large $(\bigcirc)$ and small $(\square)$ cages) extracted from spectra of Fig. 8. The large systematic errors are estimated from different types of fits.

an improved quality of the fit. The intensity ratio of the two components is fixed to $3: 1$. This is expected from the numbers of large and small cages in the cubic I clathrate structure with all cages filled by guest molecules. The intensities of the tunneling bands support this assumption. To reduce further the number of fit parameters the total quasielastic intensity was fixed relative to the elastic intensity for temperatures $T>60 \mathrm{~K}$. Below this temperature quantum behavior affects the spectra. The final fit therefore contained in general only four free parameters, an overall intensity, the widths of the two quasielastic components and an overall offset. For $T \leq 60 \mathrm{~K}$ the intensities of the elastic and quasielastic lines are fitted independently. Selected spectra (symbols) and corresponding fits (lines) are shown in Fig. 8. The fitted linewidths are shown in Fig. 7. While the statistical errors are small, there are significant systematic uncertainties. For example the results are very sensitive to the correction of the background. The background is linearly interpolated from two scans measured at temperatures of $10 \mathrm{~K}$ and $120 \mathrm{~K}$. A change of the fit regime, too, modifies the extracted linewidths seriously. Uncertainties of the relative intensities of the two quasielastic lines due to nonstoichiometry have influence on the parameters. It is these systematic errors estimated from various fit procedures which are shown in Fig. 7.

According to eqns. (4) and (5) rotational diffusion and jump reorientation are both represented by a single Lorentzian for the small momentum transfers accessible in the present experiment. In favour of rotational diffusion Fig. 7 shows a linear

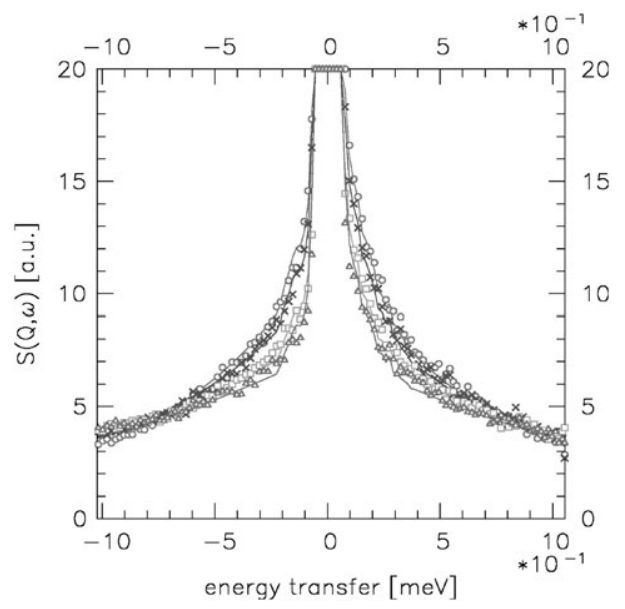

Fig. 8 Temperature dependence of the quasielastic spectra of methyl fluoride water clathrate. Fully protonated sample. Sample temperatures $T=50 \mathrm{~K}(\bigcirc)$ to $T=120 \mathrm{~K}(\triangle)$. Spectrometer: IN5 of ILL. Wavelength $\lambda=6.5 \AA$. Average momentum transfer $Q=1.6 \AA^{-1}$. Solid lines: fits. increase of the linewidths with temperature above $T \sim 50 \mathrm{~K}$ Below this temperature the shifting and broadening tunneling lines have not yet merged into the elastic line and thus cause an apparently increased linewidth. In a similar way methane clathrate shows clear features of the quantum character of rotation up to $T \sim 70 \mathrm{~K} .{ }^{22}$ Thus, the spectra can only be interpreted classically at sample temperatures larger than the activation energies. Therefore the reorienting methyl group seems not to feel the corrugation of the potential surface. That is why the description with two Lorentzian fits so well. Even for this simplified description with the many restrictions applied to reduce the number of free fitting parameters the extracted parameters scatter largely depending on details of the corrections, ranges, weights etc. It will certainly be possible to describe the spectra consistently with a dynamical model generalized to a potential distribution function $p\left(V_{3}\right)$ but it looks impossible to extract in this and similar cases of weak hindrance the underlying $p\left(V_{3}\right)$ from quasielastic spectra. The reason is probably that the timescale of the dynamics changes much more slowly with varying potential strength as in the case of strong potentials. Such potentials are present in glassy materials where the shape of the potential distribution function was taken as known and where suitable parameters could model the experiment quantitatively. ${ }^{19}$

If one bases a description on an activated Arrhenius type expression like eqn. (4) the broad linewidths of the majority species in the temperature regime $60 \mathrm{~K} \leq T \leq 90 \mathrm{~K}$ are consistent with an activation energy $E_{\mathrm{a}} \sim 7.4 \mathrm{meV}$ and a prefactor $\Gamma_{0} \sim 5 \mathrm{meV}$ which is very reasonable for methyl groups. This result will be used in the next section.

3.2.4. Excited rotational states and shape of the rotational potential. At reduced energy resolution the energy range of the IN5 spectrometer is extended to include transitions to higher rotational levels. Fig. 9 shows a doublet at about $2 \mathrm{meV}$ which is attributed to the $1 \rightarrow 2$ and $0 \rightarrow 2$ transitions. The energies are shown in Table 1 . As required they are separated by the groundstate tunnel splitting of about $0.5 \mathrm{meV}$.

The single particle model makes predictions on the excited rotational states. Results of calculations for the sharp edge of the intensity distribution at $\hbar \omega_{t}=525 \mu \mathrm{eV}$ are shown in Table 1. A comparison of these values with the spectrum of Fig. 9 shows clearly that neither a pure threefold nor a pure sixfold rotational potential can explain the observed first excited level within the SPM. Only if the dominant Fourier term of the rotational potential is of sixfold symmetry but perturbed by an $8 \%$ contribution of threefold symmetry agreement with experiment can be obtained.

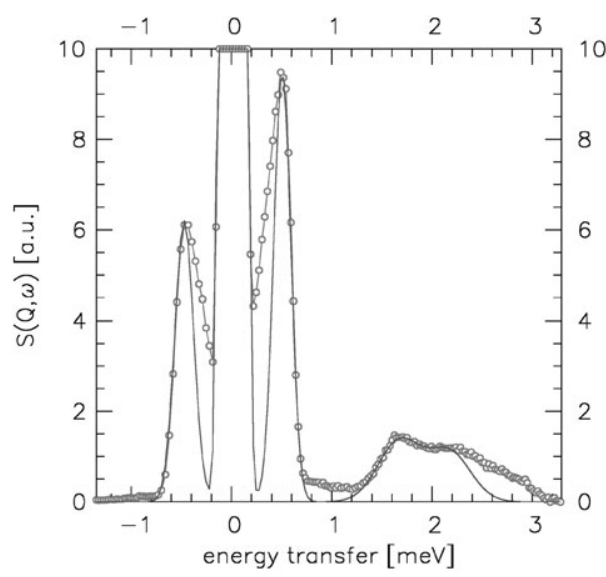

Fig. 9 Lower resolution spectrum including higher rotational levels of methyl fluoride water clathrate. Fully protonated sample. Sample temperature $T=2.0 \mathrm{~K}$. Spectrometer: IN5 of ILL. Wavelength $\lambda=4.5 \AA$. Average momentum transfer $Q=2.3 \AA^{-1}$. 
Table 1 Second excited rotational level calculated for a given tunnel splitting $\hbar \omega_{t}$ for various potentials of the single particle model and for rotation-translation coupling. For comparison the experimental results are shown

\begin{tabular}{|c|c|c|c|c|c|}
\hline Potential/meV & & $\hbar \omega_{\mathrm{t} / \mathrm{meV}}$ & $E_{0-2 / \mathrm{meV}}$ & $E_{0-3 / \mathrm{meV}}$ & $E_{\mathrm{a} / \mathrm{meV}}$ \\
\hline$V_{3}=3.15$ & & 0.524 & 3.05 & 6.06 & 1.8 \\
\hline$V_{6}=15.5$ & & 0.524 & 1.90 & 2.95 & 9.0 \\
\hline$V_{3}=0.90$ & $V_{6}=13$ & 0.525 & 2.17 & 3.38 & 7.4 \\
\hline$A_{1}=10$ & $A_{2}=0.0021$ & 0.525 & 2.08 & 4.65 & \\
\hline Experiment & & 0.525 & 2.145 & & $\sim 7.4$ \\
\hline
\end{tabular}

The shape of the potential has to be taken into account when determining a potential distribution function. We start with the function $\hbar \omega_{t}\left(V_{3}, V_{6}\right)$ for the potential $\left(V_{3}, V_{6}\right)=(0.9,13) \mathrm{meV}$ characterizing the edges of the rotational bands. Disorder may influence the potential strengths in various ways. Some limiting situations are considered: the most general case is represented by independent variations of $V_{3}$ or $V_{6}$. It may also be, however, that the potential shape is retained: $V_{3} / V_{6}=$ constant. Calculations show that at constant $V_{3}=0.9 \mathrm{meV}$ a distribution of $V_{6}$ reduces the energies of the excited rotational states 3 and 4 in disagreement with the experiment. Simultaneous changes of both, $V_{3}$ and $V_{6}$, at the condition $V_{3} / V_{6}=$ const show more weakly a similar effect for weak potential but lead thereafter to the observed increasing energy of the excited librational levels 3 and 4 . In this scenario the activation energies increase proportional to the overall potential strength $V_{3}+V_{6}$ to large values in disagreement with quasielastic scattering. A distribution of $V_{3}$ at almost constant $V_{6}$ gives the best agreement with experiment. It combines a reduction of the groundstate tunnel splitting with an increase of the excited librational levels 2 and 3 at almost constant activation energies. This latter result is in very good agreement with the successful description of the fast classical process by a single Lorentzian at all temperatures and an activation energy consistent with the calculated activation energy of $7.4 \mathrm{meV}$ (Table 1). Thus, it is largely by the asymmetry of the transition to the third rotational level that the shape of the rotational potential could be fixed.

The presence of the sixfold term of the rotational potential strongly modifies the potential distribution function calculated originally for pure $\cos (3 \varphi)$ potentials. The sixfold potential term is fixed to $V_{6}=13 \mathrm{meV}$ as the best-but oversimplifiedapproximation to our data. The variation of the tunnel splitting with an increasing strength of $V_{3}$ calculated from eqn. (2) could be very accurately modelled by a logistic function

$$
\hbar \omega_{t}=\frac{B_{2}-B_{1}}{1+\left(\frac{V_{3}}{V_{30}}\right)^{p}}-B_{2}
$$

which is differentiable for all potential values $V_{3}$. The parameters $B_{1}=-559.2 \mu \mathrm{eV}, B_{2}=13.607 \mu \mathrm{eV}, V_{30}=3.917 \mathrm{meV}$ and $p=1.852$ describe the evolution of the calculated tunnel splittings down to $20 \mu \mathrm{eV}$ really well (upper dashed curve of Fig. 4). The potential distribution function is therefore also obtained analytically from eqn. (7). Compared to the first evaluation in a purely threefold potential the maximum is shifted to a lower value $V_{3}=1.5 \mathrm{meV}$ and has a narrower halfwidth (lower dashed curve of Fig. 4).

A trivial symmetry contained in the rotational potential is that of the rotor. Additional symmetry of the adsorption site of the guest molecules may modify this symmetry. An obvious simple possibility to explain the almost sixfold potential is that the adsorption site itself has a sixfold symmetry axis. This is the case if the molecular symmetry axis coincides with the sixfold axis of the $5^{12} 6^{2}$ cage. Less obvious is the second configuration where the molecular symmetry axis looks along a twofold axis of the cage. In this case the combination of the twofold site symmetry with the threefold molecular symmetry creates a sixfold potential. Six such twofold axes lie in the plane perpendicular to the sixfold axis and at half height through the middle of two opposite hydrogen bonds of the cage (see Fig. 1). A decision between these two possibilities is based on the interpretation of the spectra from methyl iodide clathrate. ${ }^{8}$ In this material three adsorption sites and an influence of the hydrogen bond dynamics on the spin conversion times is observed. This observation could only be explained by an orientation of the guest molecule along a twofold axis. Due to the similarity of methyl iodide and methyl fluoride we adopt this interpretation for methyl fluoride clathrate. (In the meantime a proposal is accepted to measure the crystal structure of methyl fluoride clathrate to prove the above conclusion independently.)

An interesting question concerns the difference of the water cages in the cubic I and cubic II clathrate structures. No rattling mode is found in $\mathrm{CH}_{3} \mathrm{~F}$ possibly due to stronger hydrogen bonds with the fluorine. No spin conversion could be observed within the time resolution of the experiment despite the hydrogen bonds connecting hexagons with pentagons are present. These short bonds were made responsible for conversion in the case of methyl iodide clathrate. ${ }^{8}$ However, individual bonds cannot be distinguished in the continuous smooth potential distribution. Despite the larger $5^{12} 6^{4}$ cage the methyl groups of $\mathrm{CH}_{3} \mathrm{I}$ are more strongly hindered as those of $\mathrm{CH}_{3} \mathrm{~F}$ in $5^{12} 6^{2}$ cages. The stronger reduction in size of the guest molecule is the most likely reason for this shift. Another possible explanation is an increased local symmetry. In the case of methane the softer potential of guest molecules in the small cages was attributed to the higher symmetry of the smaller cage. ${ }^{17}$ Such an environment weakens or cancels the Fourier terms of low order. These questions cannot be answered on the basis of the actual data. At least a crystal structure determination of the quality of methane clathrate ${ }^{17}$ is needed for further understanding.

\subsection{Alternative explanations}

The achieved consistency of the single particle model with experiment is not a proof of the model. Therefore we consider here another established dynamical behavior: The large cage volume available in a clathrate for a guest molecule may allow coupled translation and rotation of the latter. The theory of this rotation translation coupling (RTC) is quantitatively developed. In the present case RTC can almost exactly reproduce the edge energies of the observed excited torsional state (Table 1). We exploit the effect of disorder to further test the model. If RTC is the correct description of rotational dynamics it should be able to describe the broadened tunneling bands by smooth changes of the potential parameters around the characteristic values. The Hamiltonian eqn. (6) contains two potential parameters. $A_{1}$ is characteristic of a single particle barrier, $A_{2}$ determines mainly the centre-of-mass motion. We have calculated the response of the excitation energies to small changes of both quantities. A reduced splitting of the two lowest levels is most easily obtained by increasing $A_{2}$ at 
constant $A_{1}$. The change of the relative strengths of the potential parameters influences the excentricity $\rho$ of the centre-of-mass motion according to $\rho \sim\left(A_{2} / A_{1}\right)^{1 / 2}$. However, contrary to the description by the SPM a decrease of the groundstate tunnel splitting is always connected with a decrease of the energy of the third rotational level. This is inconsistent with the observation of the sharp high energy edge of the $0 \rightarrow 1$ and the sharp low energy edge of the $1 \rightarrow 2$ transition.

An astonishing relation between the two observed rotor levels is their ratio $E_{02} / E_{01} \sim 4$. This is the ratio characteristic of a free rotor. However, the rotational constant belonging to the rotational energies differ to that of an unperturbed methyl group. A possible way to explain such sequences of apparently free rotor levels with a characteristic energy different from the literature value of the rotational constant $B$ is based on geometrical changes of the rotor by interaction with its environment. In our case a reduced rotational constant of the methyl group compresses all free rotor levels while maintaining the characteristic $J^{2}$ sequence of levels, $J$ being the rotational quantum number. At such conditions disorder of pure $\cos (3 \varphi)$ potentials creates the right asymmetry of the transition bands. Both, an increase of the distance of the methyl protons from the axis of rotation or a change of the tetrahedral $\mathrm{F}-\mathrm{C}-\mathrm{H}$ angle towards $90^{\circ}$ reduce $B$. The largest deformation of the molecule is given by a plane $\mathrm{CH}_{3}$ group. The corresponding reduction of the rotational constant by $11 \%$ can not reduce the free rotor levels sufficiently however, to make the levels coincide with the experiment. In any case it is difficult to imagine that external forces can achieve such strong deformations while keeping the rotational potential close to zero.

Rotation of ammonia adsorbed as a submonolayer on magnesium oxide ${ }^{23}$ or of methyl groups in the calix[4]arene : $p$-xylene $1: 1$ complex inclusion compound ${ }^{24}$ have been explained by RTC. In the latter material no disorder is found. The lack of a hydrogen bond network leads in this case to long range periodicity of all atoms of the structure. In the methyl fluoride clathrate this periodicity exists only for the oxygen atoms. Thus we can conclude that the availability of 2 hydrogen sites in the $\mathrm{O}-\mathrm{H} \cdots \mathrm{O}$ bond is the cause of the disorder. Consistently all inclusion compounds with a hydrogen bonded cage network like methane clathrates ${ }^{10}$ or methyl iodide clathrate $^{8}$ show broad tunneling bands. Future $a b$ initio calculations similar to those for the case of methane clathrate ${ }^{17}$ shall quantify this conclusion for the present system.

\section{Conclusion}

High resolution neutron scattering is found to give precise structural and dynamical information on guest molecules confined in water cages. The water host lattice of methyl fluoride clathrate is formed by two types of cages. The presence of 2 separated tunneling bands of different intensities shows that both cages contain $\mathrm{CH}_{3} \mathrm{~F}$ molecules. The lower intensity of the low energy tunneling band shows that the minority species fill all $25 \%$ small $5^{12}$ cages and experience the stronger rotational potential. $75 \%$ of the molecules house the $5^{12} 6^{2}$ cages and perform almost free rotation. Consistently, quasielastic spectra are perfectly described by two Lorentzians of intensity ratio 3 : 1 , the which is observed in the tunneling spectrum. However, no signs of disorder are visible in the quasielastic spectra.

The most detailed results are related to the majority $\mathrm{CH}_{3} \mathrm{~F}$ species filling the large $5^{12} 6^{2}$ cages. The combined analysis of the two lowest rotor transitions show that the dominant potential term has sixfold symmetry $V_{6}=13 \mathrm{meV}$ modified by a weak threefold term $V_{3}=0.9 \mathrm{meV}$. It is proposed that the sixfold symmetry is due to an orientation of the symmetry axis of $\mathrm{CH}_{3} \mathrm{~F}$ molecule towards a hydrogen bond as in methyl iodide clathrate. ${ }^{8}$ The combination of the respective threefold and twofold symmetries creates the sixfold potential.
Broad tunneling bands show the presence of disorder. Compared to other inclusion compounds a water clathrate is distinguished by its hydrogen bonds. The multitude of different realizations of the hydrogen bond network of a cage consistent with the ice rules are the origin of disorder. Contrary to the $\mathrm{CH}_{3} \mathrm{I}$ clathrate there is no specific influence of the potential landscape of the cage surface on the rotational potential. The effects average out to a single intensity profile. The tunneling intensity increases linearly with energy transfer and shows a sharp cut-off close to the free rotor energy. The underlying potential distribution function $p\left(V_{3}, V_{6}\right)$ has to reproduce the almost constant low activation energy observed by quasielastic spectroscopy. $p\left(V_{3}, V_{6}\right)$ is obviously centered around the above potential values with almost constant $V_{6}$ and disorder mainly concentrated in the variation of the weak $V_{3}$ term. A heuristic analytical expression of $p\left(V_{3}, V_{6}=13 \mathrm{meV}\right)$ is presented.

Rotation translation coupling observed in other cage structures can be excluded because disorder leads to the wrong asymmetry of the excited librational band.

To confirm the above results by numerical modelling a structure determination is needed. A corresponding proposal is accepted.

\section{Acknowledgements}

One of us (MPr) thanks Hans Grimm for very helpful discussions and contributions.

\section{References}

1 K. A. Kvenvolden, Rev. Geophys., 1993, 31, 173.

2 Inclusion Compounds, ed. J. L. Atwood, J. E. D. Davies and D. D. MacNicol, Oxford University Press, Oxford, vol. 5, 1991.

3 F. Vögtle, Supramolekulare Chemie, Teubner, Stuttgart, 1992.

4 J.-M. Lehn, Supramolecular Chemistry: Concepts and Perspectives, VCH Verlag Ges., Weinheim, 1995.

5 M. Nakasako, J. Biol. Phys., 2002, 28, 129.

6 E. D. Sloan, Jr., Clathrate Hydrates of Natural Gases, Marcel Dekker, New York, 2nd edn., 1998.

7 C. Gutt, B. Asmussen, W. Press, M. R. Johnson, Y. P. Handa and J. S. Tse, J. Chem. Phys., 2000, 113, 4713.

8 (a) M. Prager, J. Pieper, A. Buchsteiner and A. Desmedt, Physica, 2004, B350, E399; (b) M. Prager, J. Pieper, A. Buchsteiner and A. Desmedt, J. Phys.: Condens. Matter, 2004, 16, 7045.

9 W. Press, Single Particle Rotations in Molecular Crystals, Springer Tracts in Modern Physics, Springer, Berlin, vol. 81, 1981.

10 C. Gutt, W. Press, A. Hüller, J. Tse and H. Casalta, J. Chem. Phys., 2001, 114, 4160.

11 M. Prager and A. Heidemann, Chem. Rev., 1997, 97, 2933.

12 B. Asmussen, M. Prager, W. Press, H. Blank and C. J. Carlile, J. Chem. Phys., 1992, 97, 1332.

13 M. Bée, Quasielastic Neutron Scattering, Adam Hilger, Bristol, 1988.

14 (a) V. F. Sears, Can. J. Phys., 1966, 44, 1299; (b) V. F. Sears, Can. J. Phys., 1967, 45, 237; (c) P. Schiebel, A. Hoser, W. Prandl, G. Heger, W. Paulus and P. Schweiss, J. Phys., 1994, C6, 10989; (d) Y. P. Handa and J. G. Cook, J. Phys. Chem., 1987, 91, 6327; (e) M. Prager, Physica, 2000, B283, 376.

15 H. Schober, H. Itoh, A. Klapproth, V. Chihaia and W. F. Kuhs, Eur. Phys. J., 2003, E12, 41.

16 B. Chazallon, H. Itoh, M. Koza, W. F. Kuhs and H. Schober, Phys. Chem. Chem. Phys., 2002, 4, 4809.

17 C. Gutt, B. Asmussen, W. Press, M. R. Johnson, Y. P. Handa and J. Tse, J. Chem. Phys., 2000, 113, 4713.

18 http://www.ill.fr/YellowBook/IN5/.

19 J. Colmenero, R. Mukhopadhyay, A. Alegria and B. Frick, Phys. Rev. Lett., 1998, 80, 2350.

20 M. Prager, P. Schiebel and J. Combet, Chem. Phys., 2002, 276, 69.

21 A. C. Hewson, J. Phys., 1982, C15, 3841-3855.

22 H. Conrad, W. F. Kuhs, K. Nünighoff, C. Pohl, M. Prager and W. Schweika, Physica, 2004, B350, E647.

23 M. Havighorst, M. Prager and G. Coddens, Chem. Phys. Lett., 1996, 259, 1 .

24 P. Schiebel, G. Amoretti, C. Ferrero, B. Paci, M. Prager and R. Caciuffo, J. Phys.: Condens. Matter, 1998, 10, 2221. 